\title{
Lymphoproliferative Disease Associated with Primary Immune Disorder
}

National Cancer Institute

\section{Source}

National Cancer Institute. Lymphoproliferative Disease Associated with Primary Immune

Disorder. NCl Thesaurus. Code C150673.

A lymphoid proliferation that arises in the setting of immune deficiency due to a primary immunodeficiency or immunoregulatory disorder. The primary immune disorders most frequently associated with lymphoproliferative disorders are ataxia-telangiectasia, Wiskott-Aldrich syndrome, common variable immunodeficiency, severe combined immunodeficiency, X-linked lymphoproliferative disease, Nijmegen breakage syndrome, hyper-IgM syndrome, and autoimmune lymphoproliferative syndrome. (WHO 2017) 